Ana Sofía Hernández Serrano 


\section{El objeto común en el arte}

\section{Introducción. La idea de arte}

Cuando se piensa en arte, lo primero que se nos viene a la mente es una expresión de la actividad humana mediante la cual se manifiesta una visión personal sobre lo real o imaginado, es decir, el artista imita, expresa y crea, ya sea copiando lo que hay a su alrededor o fantaseando con lo que no hay. Pero, ¿será ésta una definición concreta? Al preguntar a varias personas qué es lo que consideran como arte, muchas contestarán que es eso que te hace sentir, eso que te hace escapar de la realidad, mientras que otros dirán que es lo que está en las galerías o museos y que ellos no entienden las obras de arte de hoy en día.

A lo largo de la historia se ha buscado dar una definición de arte; por ejemplo, se tiene la concepción de éste como la actividad elaborada del hombre por oposición a la obra de la naturaleza, es decir, aquí el arte consistía en el trabajo humano en cuanto que éste realizaba cosas que no existían por el juego de las fuerzas naturales; así mismo, está el arte como técnica especializada, como disciplina intelectual, la cual, en este sentido, interesa a la estética ${ }^{1}$; sin embargo, no se ha llegado a un concenso en absoluto. Están aquellos que tienen una visión más mimética del arte o aquellos que piensan lo contrario. Al querer definir el arte, se cae en un problema sin salida, pues si se dice que consta de ciertas características o corresponde a cierta definición, posiblemente salga una postura que diga lo contrario a lo que se planteó con anterioridad.

Ahora bien, el arte en su concepción clásica era imitación, pero ya no se puede definir como tal, pues algunas imitaciones no son obra de arte y viceversa ${ }^{2}$; tampoco se puede definir como la ex-

1 Cfr. Sourian, Étienne, Diccionario Akal de Estética, trad. Ismael Grasa Adé, Xavier Meilán Pita y otros, Akal, España, 1998, pp. 135-136.

2 Cfr. Pineda, Fabrizio, ¿Qué es una obra de arte cuando todo puede ser arte? Analizando la respuesta de Arthur Danto, Universidad del Rosario, 2010, p. 7. 
presión de emociones, porque las emociones son consideradas como un evento inferior del sujeto y por ello no sirven de criterio para discriminar entre lo que es una obra de arte y un simple objeto que exprese emoción. ${ }^{3}$ Así mismo, no puede ser entendido como un arreglo de elementos que suponen una actitud estética, como decía Kant, pues al definirlo de esta forma no se distingue entre la apreciación de una obra o el experimentar el efecto de algo. ${ }^{4} \mathrm{El}$ arte no es inefable, como propuso Wittgenstein, ya que "supone que el conjunto de obras de arte es una serie lógicamente homogénea de objetos que el público es capaz de reconocer, pero no definir." 5

\section{Una obra de arte y su modo de estar}

Fundamentados en lo anteriormente dicho, considero que hoy en día el problema que deben tratar tanto la estética como la filosofía del arte ya no es buscar una definición del arte sino hacerse esta pregunta: ¿por qué algo es una obra de arte cuando algo exactamente igual no lo es? Ya no se busca una definición, sino las condiciones para que un objeto sea considerado obra de arte, pues una definición filosófica del arte debe ser compatible con cualquier tipo de arte, por más contrarios o diferentes que éstos sean. Pienso que no puede haber una definición del arte, pues ésta debe capturar todo, y por lo tanto no excluir nada, y ¿cómo hacerlo si hay demasiados estilos de arte y éstos difieren entre ellos?

Desde el siglo antepasado se ha llegado a decir que el arte ha muerto o que al menos ha llegado a un fin, y con esto ha perdido valor para la sociedad. El primer filósofo en declarar el "fin del arte" fue G.W.F. Hegel. ${ }^{6}$ Para este filósofo el arte es una forma o nivel del Espíritu Absoluto; recuérdese que ocupaba el primer lugar, en seguida venía la religión y, por último, la filosofía. El arte, en el sistema propuesto por Hegel, ocupa el primer nivel del conocimiento del Espíritu Absoluto, puesto que se encarga de lo meramente sensible, de manifestar bajo la forma sensible y adecuada la idea que

3 Cfr. Idem.

4 Cfr. Idem.

5 Idem.

6 Cfr. Cubo Ugarte, Oscar, Hegel y el fin del arte, UNED, 2010, consultado en: https://dialnet.unirioja.es/descarga/articulo/5270983.pdf, p. 10. 
constituye las cosas, y por lo tanto, la filosofía del arte debe percibir esta idea y la manifestación de ésta en la historia de la humanidad. ${ }^{7}$ Con el arte el hombre se concibe a sí mismo, se piensa a sí mismo, reflexiona sobre su propio ser, y en esto consiste la grandeza del arte, pues éste tiene que apelar a la interioridad humana.

Así mismo, Hegel divide el arte en tres etapas: la primera es la de los griegos, que consistía en un arte-religión, el que, para Hegel, representa la unidad, ya que en este tipo de arte se tiene en conjunto la ilustración de los dioses con la forma espiritual; la segunda etapa es el cristianismo, que deja de ser arte-religión, siendo el arte el que se subordina al pensamiento; y finalmente, el arte nuevo o simbólico, al que Hegel llama romántico, y que es libre. ${ }^{8}$

El arte romántico es, para Hegel, la última etapa antes del fin del arte, pues se disuelve la unidad y el fin del arte surge como esa nostalgia por la pérdida de esa unidad, que se representa con el ideal que constituye un canon artístico. Hegel ve el arte como algo pasado, como algo históricamente construido, que ha dejado de ser lo que era, que pierde sus bases; sin embargo, éste continúa en el devenir histórico, pues es considerado como una actividad central en el ser humano. Al continuar en el devenir histórico, podemos recurrir al arte gracias a la memoria. Entonces, la propuesta de $\mathrm{He}$ gel sobre el fin del arte es algo que nos remite al pasado, pues es una actividad que se recuerda a sí misma. ${ }^{9}$

El fin del arte, para Hegel, ocurre cuando se rompe con el canon y el apego a las normas que se consideraban como una guía, y el arte se vuelve una actividad sin esencia, que ha perdido su concepto y ha quedado, de cierta manera, vacío. Sin embargo, el fin del arte, para Hegel, no es el fin de la producción posthistórica, es decir, después del fin del arte, sino que el arte toma un sentido filosófico, y por lo tanto se vuelve objeto de estudio de la filosofía; al hacer esto, deja de satisfacer las necesidades del Espíritu.

El fin del arte está condicionado por el desarrollo del Espíritu Absoluto ${ }^{10}$, que debe desligarse poco a poco de lo sensible

7 Cfr. Hegel, G.W.F., Lecciones sobre la estética, Mestas ediciones, España, 2003. pp. 279-280.

8 Cfr. Ibidem p. 86.

9 Cfr. Pérez Carreño, Francisca, ¿Pero esto es arte?, Hitos del arte reciente, 2010, p. 1.

10 Cfr. Cubo Ugarte, Oscar, Hegel y el fin del arte, op. cit., pp.10-11. 
para pasar al dominio del pensamiento. Así mismo, surge con el agotamiento de la modernidad occidental, pues en las sociedades capitalistas hacer arte se ha vuelto algo totalmente superfluo, que se muestra como lo que tiene cierta permanencia en el mundo, es decir, se hace arte sólo para incorporarse al proceso de producción y consumo de mercancías, para entrar en un circuito selecto donde no todos tienen acceso. $\mathrm{Y}$ de cierta manera, esto ha seguido estando vigente, tanto en el siglo XX como en el XXI.

Algunos de los criterios para diferenciar una obra de arte, de un objeto común, son que una obra de arte es aquella que se reconoce por algún elemento intrínseco, como su capacidad de producir emociones o comunicar sentimientos. Mientras que el otro criterio, y en el que quiero hacer énfasis, es que las obras de arte adquieren su estatus basadas en un contexto histórico o social específico. ${ }^{11}$

La teoría del mundo del arte que propone Danto, de la cual hablaré más adelante, es un claro ejemplo de este último criterio, pues el curso de la historia de las artes visuales en el siglo XX fue determinado, en gran medida, por las teorías artísticas, lo que dio como resultado la desaparición de los límites que hacen al arte, los límites entre los objetos cotidianos y los objetos que son reconocidos como obras de arte, y esto sólo puede ser explicado dentro de un contexto cultural. ${ }^{12}$

Según T. W. Adorno, en su obra Teoría Estética, en el siglo XX el arte se democratiza, alcanzando niveles muy altos de popularidad, pues en la medida en que el arte corresponde a una necesidad social, se ha convertido en un negocio mientras éste sea rentable, ${ }^{13}$ y por lo tanto pierde su función de culto y se transforma en mera mercancía o cosa; es considerado, de alguna manera, una herramienta política tendiente a mantener el statu quo. ${ }^{14}$

Lo que identifica al arte verdadero es su carácter negativo, es decir, que va en contra del sistema, y así tiene autonomía, pero al mismo tiempo también se configura como hecho social. Se configura como tal porque las nuevas condiciones sociales y los nuevos

11 Páez, Andrés, El problema de la demarcación en estética: una crítica del criterio de Danto, Revista de Estudios Sociales, núm. 29, Bogotá, 2008, p. 147.

12 Cfr. Ibidem p. 8.

13 Cfr. Adorno, Th. W., Teoría Estética, Akal, España, 2004, p. 32.

14 Cfr. Fernández, Paula. La estética desencantada de T.W. Adorno. Crisis de la apariencia, op. cit., p. 44. 
medios de producción cambian la forma en que se produce el arte. Pienso que se podría decir que en el siglo XX hemos vivido en un mundo donde la meta a la que siempre se quiere llegar es satisfacer las necesidades de consumo, a adquirir mercancías y, al hacer esto, otorgar más dominio a los grupos que están en el poder. En ocasiones, algunos han intentado ir en contra de esto, sin embargo, tal vez sin darse cuenta, esos grupos "rebeldes" caen de nuevo en esta sociedad de consumo. El arte, por lo tanto, se convierte en un objeto más del que nos servimos para satisfacer a la sociedad. Con las vanguardias, las categorías del paradigma estético tradicional se dejan de lado. Un ejemplo de esto es el Pop Art, que surge como una respuesta en contra del arte elitista al poner en alto el valor de lo cotidiano a través de objetos cotidianos.

De alguna manera, el arte se niega, según Adorno, a ser definido ${ }^{15}$, pues "el arte tiene su concepto en la constelación de momentos que va cambiando históricamente" ${ }^{16}$, es decir, tanto la definición como el proceso de producción cambian; no es lo mismo el arte en la Grecia antigua que el arte que se llevaba a cabo en el siglo XX o incluso por las vanguardias, el propósito de éstas, de hacer arte, ya no encaja en nuestra sociedad, porque el contexto ya no es el mismo.

Ahora bien, con el fin de la modernidad y la llegada de la posmodernidad, el campo de la creación artística se ha vuelto totalmente permisivo y cualquier cosa puede tomarse como arte, incluso un objeto de uso común; un ejemplo claro es la Brillo box de Andy Warhol, a la que Danto recurre constantemente en su obra. ${ }^{17}$

\section{Lo contemporáneo del arte}

Para Danto, la idea del fin del arte representa un cambio en la manera de hacer las cosas y no que se dejen de hacer obras de arte, pues éstas son necesarias para que pueda seguir habiendo crítica de arte. No puede juzgarse si el arte es bueno o malo, porque se ha emancipado de las normas que limitaban la práctica artística, se ha

15 Cfr. Adorno, Teoría Estética, op. cit., p. 10.

16 Fernández, Paula, La estética desencantada de T. W. Adorno. Crisis de la apariencia, op. cit., p. 44.

17 Esta pieza es tomada como ejemplo en "El mundo del arte", "La transfiguración del objeto común", "El abuso de la belleza", entre otras. 
pasado a una etapa donde todo está permitido; prueba de ello es el arte pop o el arte conceptual, que muestran que ya no es necesaria la presencia de un "objeto extraordinario" totalmente identificable para que pueda hablarse de arte, sino que ahora cualquier cosa puede ser arte, no hay límites. Entonces, para Danto el fin del arte es una liberación total de la forma de producir arte, pues se dejan de lado las reglas establecidas durante la era antes del fin del arte, donde todo está permitido y ese arte se ha vuelto exclusivo para quienes forman parte del circuito del arte. ${ }^{18}$

En el siglo XX, el "artista pintaba sus sentimientos y no algo visible"; ${ }^{19}$ no pintaba algo idéntico a la naturaleza, que en un principio era considerado lo más bello, sino una interpretación de ésta. Ahora, en este mismo siglo, lo que se intentaba hacer era llevar la realidad al arte, cuando en un principio la realidad era aquello que el arte representaba. A partir de esto, se dice que todo, cualquier cosa, podía convertirse en arte, pero eso no significa que todo sea arte. ${ }^{20}$ Danto decía que la lógica de la historia del arte realmente hacía que pareciera que el arte es un concepto abierto, ${ }^{21}$ pues muchos artistas optaron por rechazar la idea tradicional de arte, así como también los materiales tradicionales para llevarlo a cabo, y en su lugar empezaron a utilizar cualquier cosa, como los materiales que empleamos en la vida cotidiana.

Ante esta situación, es decir, la necesidad de distinguir entre el arte y las cosas reales que no lo son, se puede tener como criterio el gusto, que fue el concepto central en la estética del siglo XVIII. El problema central de éste se divide en dos opiniones, por decirlo de alguna manera: la primera es que sobre los gustos no se discute, y la segunda dice que existe el buen gusto y que, por lo tanto, el gusto no es tan subjetivo como lo hace ver la primera posición. Por otro lado, está el criterio de la belleza, que es considerada una de las muchas cualidades que tiene la obra artística. ${ }^{22}$

18 S/a, Fin del arte según Arthur C. Danto, p. 3.

19 Danto, Arthur C, Qué es el arte, traducción de Iñigo García Ureta, Paidós, España, $3^{a}$ edición, 2013, p. 29.

20 Cfr. Ibidem p. 42.

21 Cfr. Ibidem p. 49.

22 Cfr. Cataldo Sanguinetti, Gustavo. El esplendor de la idea: belleza y apariencia en Hegel, Revista de humanidades vol. 15-16, junio- diciembre, Santiago, Chile, 2007, p. 6. 
Ahora bien, la belleza es vista como una cualidad sensible, pero existen casos en que algunas personas no poseen ninguna sensibilidad en cuanto a la belleza de algo, y por lo tanto no la consideran importante. Arthur Danto, en su libro El abuso de la belleza, distingue dos tipos de belleza: la interna y la externa. La segunda, la externa o estética, es aquella que tiene que ver con los sentidos $^{23}$, es decir, es aquello que tachamos de feo o hermoso de manera inmediata, por decirlo de alguna manera. Este tipo de belleza es, pues, física. Por otro lado, la belleza interna o artística es aquella que "requiere percepción e inteligencia critica" ${ }^{24}$, es decir, es parte del significado de la obra. Entonces, si tomamos esta diferenciación del concepto de belleza, podemos decir que ésta, entendida como artística o interna, es más que la pura emoción óptica, ya que puede formar parte del significado de la obra. La belleza interna está ligada a un contenido, está cargada de significado y es creada por el hombre. ${ }^{25} \mathrm{La}$ apariencia, la forma de las obras de arte, no es lo principal, sino el significado.

No es posible definir el arte, o mejor dicho distinguir entre una obra de arte y un objeto común, mediante criterios meramente perceptivos, puesto que éstos pueden ser perceptivamente iguales, indiscernibles. Dado lo anterior, la obra de arte se convierte en un ejercicio de pensamiento $y$, por lo tanto, necesita de dos rasgos de carácter filosófico para demarcarla: una obra de arte es considerada como tal si es sobre algo y, como se dijo antes, si tiene un significado y lo encarna. ${ }^{26}$

El primer rasgo que caracteriza al arte es la representación sobre un objeto ${ }^{27}$. Sin embargo, esto no puede ser criterio suficiente para caracterizar el arte como tal, ya que, en principio, todo tipo de representaciones, ya sean artísticas o no artísticas, satisfarían esta condición. Lo que distingue a las obras de arte de otras representaciones es el modo en el que las primeras son sobre algo,

23 Cfr. Danto, Arthur. El abuso de la belleza. La estética y el concepto de arte, Paidós, Barcelona, 2005, p. 141.

24 Ibidem, p. 142.

25 Cfr. Carrasco Barranco, Matilde. De la estética de la forma a la estética del significado. Sobre el giro estético de A. Danto, Revista de Filosofía, vol. 38, núm. 1, 2013, p. 92.

26 Cfr. Danto, Arthur. ¿Qué es el arte?, op. cit., p. 147.

27 Cfr. Idem. 
son sobre su objeto. La obra de arte debe ser sobre algo, expresar un punto de vista hacia ese tema que es el que buscan transmitir a sus receptores, y eso es lo que distingue una obra de arte de un objeto común ${ }^{28}$, ésta no tiene que representar algo en un sentido estricto, sino que establece una serie de relaciones entre objetos y representaciones.

El segundo rasgo, y con el cual se deja de lado la ambigüedad del primero, es que las obras "encarnan" su significado ${ }^{29}$, es decir, el significado encarnado es el significado de una obra dada y alude al modo en que ese significado se encarna en el objeto material que la transporta ${ }^{30}$ Una obra de arte es, pues, algo que encarna aquello que representa, y al hacerlo, ésta misma se convierte en el objeto de referencia, adquiere su autonomía y deja de depender de la filosofía para determinar su propia naturaleza, pues, desde las vanguardias "la obra de arte adquiere una veneración por sí misma"31 es decir, es dueña de su significado. Ahora lo que el espectador debe hacer es interpretar las prioridades que provee el significado, de tal manera que llegue a comprender el significado esperado que encarnan. ${ }^{32}$

Así mismo, para Danto la idea estética es aquella a la que se ha dado un conocimiento sensible, pues la obra de arte es elaborada por alguien, el artista, y éste vive en cierto contexto histórico, y es aquí donde pienso que la obra nos permite conocer, por decirlo de alguna manera, ya que ésta es parte de ese contexto, es parte o muestra de éste, y por medio de ella se puede llegar a conocer cierta época.

Uno de los momentos más radicales en el arte se dio a partir de las vanguardias, pues con éstas se marcó una ruptura respecto del arte tradicional. Fue en esa época en la que la concepción de arte dio giro tras giro debido a las nuevas corrientes que se llevaban a cabo con el fin de reivindicar al arte, pues creían que el arte no se podía regir, como lo dice Gombrich, sólo por la fidelidad. Por ejemplo, con el impresionismo se dio lo que se conoce como el fin

28 Cfr. Carrasco Barranco, Matilde. De la estética de la forma a la estética del significado. Sobre el giro estético de A. Danto, op. cit., p. 85.

29 Cfr. Danto, Arthur. ¿Qué es el arte?, op. cit., p. 147.

30 Cfr. Carrasco Barranco, Matilde. De la estética de la forma a la estética del significado. Sobre el giro estético de A. Danto, op. cit., p. 93.

31 Ruiz López, Juan Ignacio, La obra de arte: objeto simbólico y mercancía, Imafronte, núm. 18, 2006, p. 109.

32 Cfr. Op. cit., Danto. Qué es el arte, p. 52.

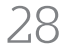


de la pintura ${ }^{33}$, pues se dejó de lado el interés de los artistas en la representación, pero también se presentó una concepción nunca antes vista del arte.

Con el impresionismo ya no hay necesidad de tener en cuenta a la representación como tal, sino a los medios de representación, es decir,"lo importante ya no consiste en lo representado sino en la forma en que es percibido por nosotros" ${ }^{34}$, la forma en la que la obra nos afecta.

A partir del impresionismo la pintura revoluciona, pues se dejan de lado, como bien dijimos antes, los temas de la composición, y en lugar de eso, ahora lo importante es la reflexión sobre la obra, por lo que, con este movimiento, el arte se vuelve su propio objeto, ya no es un medio de representación, sino su propio fin. Sin embargo, con esta revolución nace un problema: ¿cómo identificar cuál o qué es una obra de arte?

Danto identifica dicho problema con el nacimiento del Pop Art y en concreto con la obra de Warhol, pues tiene la particularidad de apropiarse de un objeto común y trasladarlo al mundo del arte. Para poder tratar este tema, Edgar Vite, más que definir, nos dice las características del Pop Art, que retoma el modelo de la producción en serie y la industrialización; por esto mismo, y la época en la que surgió, apela a un estilo de vida. Además, presenta la producción masiva como un modo efectivo de comercialización y como consumo artístico, ya no como una reflexión.

Así, la aparición de los ready-mades es entendida por el autor como un intento de Duchamp por retomar la estructura y configuración de los medios de producción, y al mismo tiempo la incorporación de los diversos elementos que rodean la vida cotidiana; sin embargo, para el artista no era sólo incorporar dichos elementos por incorporarlos con el fin de que el espectador los identificara, sino incorporar una serie de elementos que no correspondieran con las obras de arte de esa época; no buscaba que el espectador se sintiera identificado, sino todo lo contrario.

El Pop Art tomó la apariencia como su tema central, y este interés responde a que la sociedad de esa época se preocupaba por la vida lujosa, los escándalos de celebridades, etcétera; así, es clasificado por Vite como un arte exhibicionista que reclama ser con-

33 Cfr. Vite, Edgar. Marcel Duchamp y el fin de las artes figurativas, ITAM, p. 23. 34 Ibidem, p. 25. 
templado y admirado, es en pocas palabras lo que todo el mundo ve y consume. Para Danto, el Pop Art se caracterizó por cambiar de ámbito una serie de factores pertenecientes a la esfera comercial y trasponerlos a la esfera artística, y con esto, llevó a cabo una recontextualización para poner énfasis en el objeto. Así,"el arte se vuelve un juego constante entre el creador y el público", 35 al sacar objetos de su contexto y presentarlos como arte, al dejar de tomar en cuenta la finalidad del objeto para así tener una experiencia nueva sobre el mismo. El Pop Art no hace referencia a la imaginación, sino a la presencia, en el sentido de cómo se nos muestran las cosas en el mundo, por lo que terminó de separar la belleza de las obras al confundirlas con la realidad.

\section{Conclusión}

Así, los ready-mades cuestionan el concepto de reproducción y autenticidad. En éstos se elimina al artista como parte del proceso creativo; la producción no tendrá un lugar prioritario y, en esa medida, parece que el autor tiene un rol pasivo con respecto a la obra de arte. El ready-made generó nuevas condiciones, en las que la experiencia visual o retina queda suplantada por la presentación de un objeto común y corriente.

35 Ibidem, p. 36. 